\title{
Quantum Dots-Based Nano-Coatings for Inhibition of Microbial Biofilms: A Mini Review
}

\author{
Eepsita Priyadarshini, Kamla Rawat and \\ Himadri Bihari Bohidar \\ Additional information is available at the end of the chapter \\ http://dx.doi.org/10.5772/intechopen.70785
}

\begin{abstract}
Infection of implants by microbial biofilm is chiefly caused by Staphylococci, Pseudomonas and Candida species. The growth of microbes by forming biofilms offers them protection from antibiotics, drugs and host defense mechanisms. The eradication of biofilms from implants and medical devices is difficult because of the protection by the biofilm forming pathogenic microbes. Hence, researches are focused on development of antibiofilm materials, which are basically constituted of antimicrobial substances or antimicrobial coatings. Nanomaterialbased coatings offer a promising solution in this regard. Quantum dots (QDs) are the group of semiconductor nanoparticles with high photoluminescent properties compared to conventional organic fluorophores. Thus, drug-conjugated QDs can be a promising alternative for biofilm treatment, and these can serve as excellent alternatives for the mitigation of recalcitrant biomaterial-associated infections caused by resistant strains. Furthermore, their use as antibiofilm coating would avoid the dispersion of antimicrobial agents in the surrounding cells and tissues, thereby minimizing the risks of developing microbial resistivity.
\end{abstract}

Keywords: quantum dots, microbial biofilms, fluorescence, infections, antibiofilm materials

\section{Introduction}

Quantum dots (QDs) represent a class of colloidal semiconductor nanocrystals having fluorescent properties that absorb photons at a particular (lower) wavelength and emit at a higher wavelength. These QDs are basically composed of a core and corona layer. The photoluminescence emission wavelength of QDs is directly proportional to its size. The core of the QDs may contain one or more heavy elements such as cadmium, selenium, zinc or tellurium. QDs possess significant superiority over the conventional fluorophores 
in terms of physicochemical and fluorescent properties. The distinguishable fluorescent properties, smaller size, photostability, resistivity to metabolic degradation and capability of conjugation to ligands/biomolecules make QDs a superior choice for biological applications compared to conventional fluorophores.

In the last three decades, several microbes (fungi, yeast and bacteria) have emerged as major human pathogens and have been responsible for causing life threatening diseases especially in immunecompromised individuals and patients with serious medical issues [1]. The widespread and prolonged use of antifungal agents and drugs for treating the infection caused by the pathogens has resulted in increasing incidences of multidrug resistance (MDR). Additionally, several mutant strains have developed that show high resistance to the antifungal drugs being used [1]. For example, Candida albicans, a dimorphic opportunistic pathogen, occurs as a normal commensal in humans but becomes pathogenic in immunecompromised individuals. The azole resistive clinical isolates of $C$. albicans result in cross-resistance to several unrelated drugs and this arises because of the phenomenon of multidrug resistance (MDR) [2, 3]. Similarly, Pseudomonas aeruginosa and Staphylococcus aureus are the two most pathogenic bacteria known to cause severe infection and biofilm formation [4]. Several mechanisms are responsible for development of MDR, some of which involve an overexpression of drug efflux pumps encoding genes such as CDR1 and CDR2 belonging to ATP-binding cassette $[2,5,6]$; overexpression of the drug and MDR1 belonging to the major facilitator superfamily transporters $[3,6]$ and overexpression of mutations in ERG11 and encoding the target enzyme of azoles, lanosterol 14 $\alpha$-demethylase [7]. Hence, microbial infection has become a major problem with concerns focusing on those that have become resistant to antibiotics. Around 2 million people are affected annually with antibiotic-resistant bacteria of which approximately 23,000 people die as per the studies of U.S. Center for Disease Control and Prevention [8].

Microbial communities adhere to a solid surface especially in surface/water interference forming biofilms [9]. Microbes attach to the surface by means of extracellular polymeric substances (EPS), and this acts as their survival means against harsh environmental conditions. Biofilm formation is however associated with surface deterioration and corrosion. In addition, pathogenic microbes form biofilms on medical devices and implants, and this has become a great concern in the arena of healthcare. Biofilm also enhances microbial activity and provides protection against harsh environmental conditions such as drugs, antibiotics and common sanitizers. Because of the emerging conditions of MDR, there is a demand for developing new drugs, antimicrobial agents and modifiers capable of inhibiting microbial growth and biofilm formation. With the necessity of developing antimicrobial agents with diverse functionality and ability to kill both strains of bacteria, nanomaterials have been widely investigated in this regard. Silver nanoparticles [10], copper oxide nanoparticles [11-13], metal oxide nanoparticles [12, 13] and even carbon nanomaterials [14] have been reported for their excellent antimicrobial efficiency. Among these, silver nanoparticles have been extensively used as antimicrobial and antibiofilm agents due to their broad spectrum antimicrobial activity, multiple cellular targets and minimum host toxicity. However, high concentration of silver is toxic to humans and its persistent use causes argyrosis and argia $[15,16]$. Hence, the demand is for exploring novel nanomaterials with effective antimicrobial and antibiofilm properties along with biocompatibility. Therefore, the requirement must be 
targeted towards exploring novel biocompatible nanomaterials with effective antibiofilm and optical properties. QDs can be suitable alternatives because of their intriguing optical, fluorescence, high quantum yield, photostability and easy conjugation efficiency. QDs easily attach to microbial surface because of their small size and their dispersion stability is basically governed by colloidal theory [17]. These are excellent candidates in biomedical applications such as imaging, diagnosis and sensing and drug discovery. Developing QDs-based nanocomposites as coating materials on implants and catheters can thus combat pathogenic invasion and biofilm formation. QDs could be engineered with coating agents and conjugated with bioactive ligands or biorecognition elements for targeted treatment, biofilm visualization, and inhibition.

\section{Biofilm formation, its mechanism and transmission}

Biofilm can be defined as microbial cells enclosed in an exopolysaccharide matrix and adhered to a cell surface. Formation of biofilms by bacteria and fungus is a defense strategy for protection from environment. Microbes secrete extracellular polymeric substances (EPS) that act as a primary scaffold for attachment to solid substrate [18] and its basic constituents are proteins, polysaccharides, nucleic acids with some lipids and humic substances [19]. Three-dimensional study of the EPS layer suggested that it forms a gel-like network wherein microbes are embedded and it also maintains the attachment of bacteria to the solid substrate [20]. Stability to the 3D structure of EPS is rendered by the hydrophobic interactions as well as van der Waals attraction between amino acids/peptides and cations such as $\mathrm{Ca}^{2+}$ and $\mathrm{Mg}^{2+}$ [21]. Biofilm formation and its structure depend on the environmental conditions to which the bacteria are exposed. When cells are in a nutrient stress condition, an increase in EPS secretion occurs, which promotes hydrophobic interactions to allow attachment to solid substrate [22]. It has been suggested that the presence of a high concentration of EPS negatively affects the diffusion of lipophilic compounds (such as sanitizers, antibiotics and hydrocarbons), across the microbial cell surface [23, 24].

Among bacteria, P. aeruginosa is an opportunistic pathogen that causes a number of infections in humans. It develops resistance to antibiotics by forming biofilm matrices that comprise polysaccharide-EPS. It has been noticed that it forms and regulates biofilm via quorum sensing mechanism and therefore most of the researches have focused on disrupting the quorum sensing pathway [25]. Similarly, biofilm formation in Staphylococcus epidermidis has also been analyzed using different methods such as microtiter plate, congo red agar plate test and via molecular detection of the ica locus [26-28]. It was found that production of a slimy substance assisted in forming biofilm and was associated with virulence also. The production and formation of biofilm depend on the media constituents; however, the exact mechanism behind the formation of a mature biofilm is still being investigated. However, on the basis of in vitro experimental models, biofilm formation can be segregated into four different stages: (i) attachment of microbial cells to surface, (ii) formation of multi-layer structure via the accumulation and aggregation of cells, (iii) maturation of biofilm and (iv) detachment of cells from biofilm into planktonic state and initiation of a new biofilm cycle $[29,30]$. The initial step of attachment is normally driven by hydrophobic, electrostatic and Lifshitze-van der Waals forces, and hence is nonspecific in 


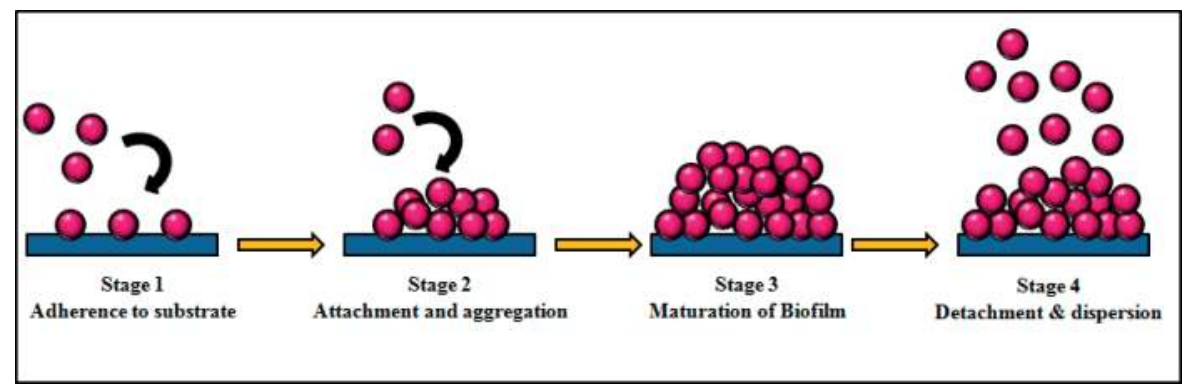

Figure 1. Schematic illustration of the different stages involved in biofilm formation and detachment.

nature. Additionally, certain specific proteins also assist in binding of the microbes to the surfaces [30]. The second step of accumulation is mediated via microbial surface components that recognize adhesive matrix molecules and occurs via an active process. This step involves the establishment of biofilm on the microbial surface. This process is followed by the maturation step. In this step, the characteristic features of the biofilm are formed on basis of specific microbial type. In the final step, where a new phase of invasion is initiated involves the detachment and dispersion of the microbes [31,32]. Figure 1 shows the schematic illustration of the different stages in the cycle of biofilm formation and detachment.

P. aeruginosa, Vibrio cholerae and some mycobacterial species are the common human pathogens that form biofilms and hence have the possibility of infecting humans. There are several mechanisms via which pathogenic microbes in the biofilm can initiate an infection. The seeding dispersal of a large number of pathogenic cells is one of the possible mechanisms that can initiate an infection, as the microbes are not sessile in a biofilm and hence can easily detach and initiate an infection. Secondly, the virulent phenotypes present in a biofilm can expand their colony and initiate infection. This is highly possible as biofilm has a huge heterogeneity in its phenotypical constitution $[33,34]$. In addition to these, several other mechanisms have been hypothesized that could possibly allow the survival of a pathogenic organism and its transmission. For example, the detachment of pathogenic microbes from the biofilm, quorum sensing [35], co-aggregation and auto-aggregation [35, 36], modification in biosynthesis of EPS and metabolic pathways and genetic mutations [37] are important issues. However, the complete understanding of the mechanism of biofilm formation and virulence requires complete analysis of the pathogen's life cycle, environmental parameters and the different phenotypes.

\section{Role of QDs in inhibiting biofilm formation}

Biomedical implants are a necessity in modern health care; biofilm formation on these implants and devices is a major cause of their failure. Mostly S. epidermidis and S. aureus are observed in contaminated biomedical implants and devices [38]. Biofilms formed on implants and medical devices are difficult to remove as they are protected by exopolymeric matrix secreted by the pathogenic microbe [39]. Although a number of metal and their nanosize 
forms (silver, copper, gold etc.) have been used as antimicrobial agents, there efficiency is diminishing due to MDR. Investigations on the antibacterial and antifungal property of QDs have been conducted, which suggests that they can serve as excellent candidates for biomedical applications because of their solubility and biocompatibility.

Aqueous solubility and compatibility make graphene quantum dots (GQDs) useful in biomedicine. GQDs are reported to be biocompatible at cellular levels investigated via WST-1 assay, LDH production, ROS generation and in vitro and in vivo distribution [40]. GQDs also possess antibacterial property against Escherichia coli and S. aureus, and GQDs with low dose of $\mathrm{H}_{2} \mathrm{O}_{2}$-based band-aids have also been prepared based on the peroxidase-like property of these particles. The designed band-aids showed a good anti-disinfectant property. They analyzed the effect of formed GQDs on biofilm formation and destruction and observed a reduction in biofilm formation by $S$. aureus at $100 \mu \mathrm{g} / \mathrm{mL}$ and $100 \mathrm{mM}$ of GQDs and $\mathrm{H}_{2} \mathrm{O}_{2}$ concentration, respectively. Furthermore, they also observed that GQDs alone also showed antibiofilm properties [41]. The studies thus suggested that appropriately designed GQDs had the ability to breakdown existing biofilms and simultaneously prevented the formation of new ones. Habiba et al. suggested the antimicrobial property of silver-graphene quantum dots against $P$. aeruginosa and $S$. aureus. They observed a synergistic effect between silver nanoparticles and GQDs with 25 and $50 \mathrm{~g} / \mathrm{ml}$ of silver-graphene quantum dots inhibiting S. aureus and $P$. aeruginosa growth, respectively. Thus, the potential applicability of Ag-GQDs as fabrication and antibacterial coating agents was clearly established [42].

Furthermore, the use of semiconductor QDs will allow visualization of biofilm inhibition due to their fluorescent properties. The current methods being used for biofilm analysis are SEM, AFM, MRI and Raman spectroscopy that require lengthy and costly procedures apart from sample modulation, which sometimes provide partial details of the samples concerned [43,44]. Other than this, conventional fluorescent dyes conjugated with carbohydrate recognition elements are used for biofilm analysis via confocal laser microscopy [45]. However, the use of a synthetic complex is sometimes toxic to cells thereby preventing in situ analysis. Therefore, QDs can be an exceptional solution for this. Moreover, amphiphilic carbon dots (CDs) have been shown to penetrate the EPS layer of $P$. aeruginosa, allowing direct visualization of its architecture, growth and how external agents affect its inhibition. The hydrocarbon side chains of CDs dock to the EPS network resulting in making the EPS scaffold highly fluorescent [46]. In yet another study, QDs with two varied surface chemistry [-COOH and polyethylene glycol (PEG) modified] were analyzed for their mobility and distribution in P. aeruginosa PAO1 biofilms. It was inferred that the QDs did not penetrate the bacterial cell but did colocalize with EPS matrix of the biofilm. While surface functionalization and QDs flow rate did not show any distinctive difference, analysis of center of density suggested that QDs with - $\mathrm{COOH}$ surface groups diffused easily compared to PEGlyated QDs. Biofilms treated with PEGlyated QDs had rough polysaccharide layers and cell distribution compared to $-\mathrm{COOH}$ functionalized QDs. It was thus concluded that treatment with nanomaterials can result in varying the structural parameters of biofilm [47]. The fluorescent property of QDs would thus allow recognition of biofilm formation at different growth stages and environmental conditions. Additionally, spectroscopic analysis can also be performed, which would allow better understanding of the phenomenon of binding of QDs to EPS. Conjugated QDs have also been used for biofilm imaging analysis. In a study, CdTe 
QDs tagged with Concanavalin A for labeling the saccharide molecules on the surface of C. albicans was studied. It relied on the ability of Concanavalin A to specifically bind to $\alpha$-D mannose and glucose residues of saccharides. They observed that almost $93 \%$ of cells were labeled with the modified CdTe particles and were highly specific in activity [48]. Similarly, CdSe/ZnS QDs surface capped by 3-mercaptopropionic acid (MPA) and the amino acids (leucine or phenylalanine) were also used for labeling the biofilm produced by Shewanella. Amphiphilic core/shell $\mathrm{CdSe} / \mathrm{ZnS}$ QDs were used for labeling the hydrophobic microdomains of biofilm produced by Shewanella oneidensis, a Gram-negative bacteria. It was inferred that CdSe/ZnS@dihydrolipoic acid-Leu or CdSe/ZnS@dihydrolipoic acid-Phe QDs showed increased hydrophobicity in comparison to CdSe-core QDs capped with 3-mercaptopropionic acid (MPA). Thus, the functional group on QD surface and the ligand density played an integral role in interaction with biofilm matrix. While the hydrophilic MPA-capped QDs were homogeneously associated, DHLALeu and DHLA-Phe QDs were specifically confined assisting in identifying the hydrophobic microdomains of biofilm. Hence, appropriate conjugation of surface functional groups can significantly dictate their interaction with biofilm [49]. Quite recently, selenium nanoparticles have been reported for their tremendous potential in biofilm inhibition in C. albicans. For the study, selenium nanoparticles were synthesized via laser ablation method and were used to analyze biofilm inhibition. They observed a very good attachment of selenium nanoparticles to the Candida surface, which was due to electrostatic attraction between the positively charged surface of Candida and negatively charged Se nanoparticles. The particles affect the cellular morphology of the fungus by substitution of sulfur groups of amino acids by the Se particles. This consequently altered the protein structure and damaged Candida morphology. Size and crystallinity of particles had a significant effect on biofilm inhibition [50].

Figure 2 presents the mode of action of quantum dots. The application of QDs as antibiofilm agents can inhibit microbial biofilm at two stages. It can act at the initial stage, where its presence would hinder further attachment of microbial cells to the solid substrate thereby preventing the progression to mature biofilm stage and EPS secretion. Secondly, QDs can act on the matured biofilm, where its penetration into the cells would result in killing of the microbes and subsequent dispersion of the formed biofilm.

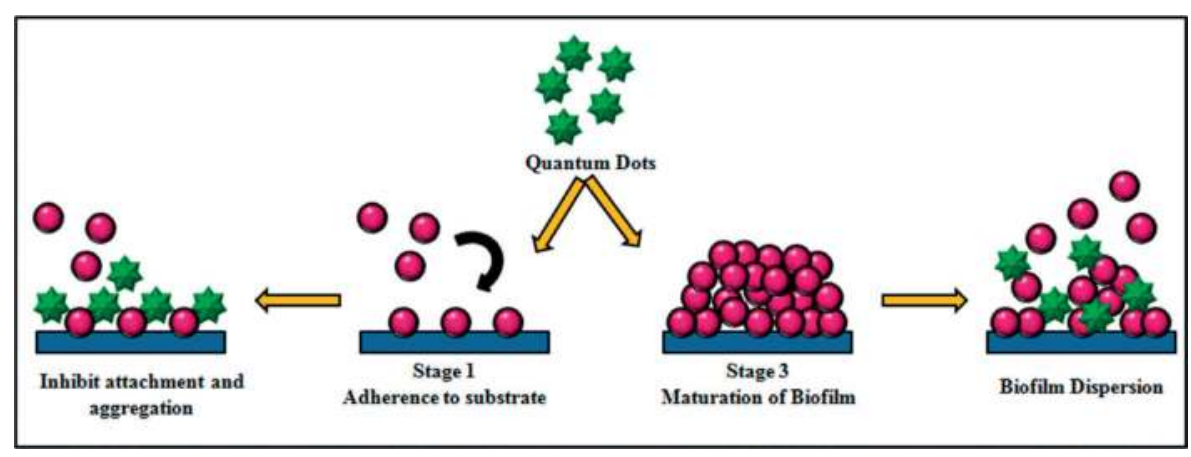

Figure 2. Schematic illustration showing the possible mode of action of antimicrobial quantum dots on biofilm. 
With this, we envision that QD-based antibiofilm coatings can be promising probes in investigating biofilm imaging, treatment and their eradication. Furthermore, their broad spectrum activity and minimal host toxicity are additional advantages in this regard. Hence, the use of semiconductor QDs would not only allow detecting the inhibition process but also favor their visible monitoring.

\section{Conclusion and future perspective}

There is a steady increase in the use of QDs. Despite the several advantages offered by QDs, with some improvements, these can emerge as excellent probes for biological applications. Focus should be towards improved protocols for functionalizing the surface of QDs simultaneously making sure that its properties remain unaltered and secondly, appropriately modifying the surface of QDs so that they do not aggregate in a protein-rich solution or cystol. These methods along with the said advantages would assist in utilizing QDs for biological and biomedical applications. Furthermore, the QDs can be tagged with antimicrobial drugs or drugs can be encapsulated inside the QD core thereby increasing the potency of drugs even at low concentration. Synergistic effect of silver nanoparticles with antibiotics such as penicillin $\mathrm{G}$, amoxicillin, erythromycin, clindamycin and vancomycin is known. Therefore, studies on the synergism between QDs and drug molecules have to be analyzed in detail. This would also assist in providing insights into the molecular mechanism of action of QDs and any kind of cellular changes occurring in the pathogen upon its interaction with pathogenic microbes. Additionally, QDs labeling would allow a high throughput analysis of biofilm inhibition and disruptions that will have significant effect in healthcare sector to identify and combat biofilm formation and pathogenic infections.

\section{Acknowledgements}

This research was supported by DST-PURSE-II funding. KR acknowledges the receipt of a DST-Inspire Faculty award from Department of Science and Technology, Government of India.

\section{Author details}

Eepsita Priyadarshini ${ }^{1}$, Kamla Rawat ${ }^{2,3}$ and Himadri Bihari Bohidar ${ }^{1,2 *}$

*Address all correspondence to: bohi0700@mail.jnu.ac.in

1 School of Physical Sciences, Jawaharlal Nehru University, New Delhi, India

2 Special Centre for Nano Sciences, Jawaharlal Nehru University, New Delhi, India

3 Inter University Accelerator Centre, New Delhi, India 


\section{References}

[1] Barelle CJ, Priest CL, MacCallum DM, Gow NA, Odds FC, Brown AJ. Niche-specific regulation of central metabolic pathways in a fungal pathogen. Cellular Microbiology. 2006 Jun 1;8(6):961-971

[2] Franz R, Kelly SL, Lamb DC, Kelly DE, RuhnkeM, Morschhäuser J. Multiple molecular mechanisms contribute to a stepwise development of fluconazole resistance in clinical Candida albicans strains. Antimicrobial Agents and Chemotherapy. 1998 Dec 1;42(12):3065-3072

[3] White TC, Marr KA, Bowden RA. Clinical, cellular, and molecular factors that contribute to antifungal drug resistance. Clinical Microbiology Reviews. 1998 Apr 1;11(2):382-402

[4] Bowler PG, Duerden BI, Armstrong DG. Wound microbiology and associated approaches to wound management. Clinical Microbiology Reviews. 2001 Apr 1;14(2):244-269

[5] Kohli A, Smriti NF, Mukhopadhyay K, Rattan A, Prasad R. In vitro low-level resistance to azoles in Candida albicans is associated with changes in membrane lipid fluidity and asymmetry. Antimicrobial Agents and Chemotherapy. 2002 Apr 1;46(4):1046-1052

[6] Stoldt VR, Sonneborn A, Leuker CE, Ernst JF. Efg1p, an essential regulator of morphogenesis of the human pathogen Candida albicans, is a member of a conserved class of bHLH proteins regulating morphogenetic processes in fungi. The EMBO Journal. 1997 Apr 15;16(8):1982-1991

[7] Prasad T, Saini P, Gaur NA, Vishwakarma RA, Khan LA, Haq QM, Prasad R. Functional analysis of CaIPT1, a sphingolipid biosynthetic gene involved in multidrug resistance and morphogenesis of Candida albicans. Antimicrobial Agents and Chemotherapy. 2005 Aug 1;49(8):3442-3452

[8] US Department of Health and Human Services, Centers for Disease Control and Prevention, Antibiotic Resistance Threats in the United States, 2013. US Centers for Disease Control and Prevention. 2013. Available from: http://www.cdc.gov/drugresistance/threat-report-2013/pdf/ar-threats-2013-508.pdf [Accessed: Jan 24, 2015]

[9] Costerton JW, Lewandowski Z, Caldwell DE, Korber DR, Lappin-Scott HM. Microbial biofilms. Annual Reviews in Microbiology. 1995 Oct;49(1):711-745

[10] Pal S, Tak YK, Song JM. Does the antibacterial activity of silver nanoparticles depend on the shape of the nanoparticle? A study of the Gram-negative bacterium Escherichia coli. Applied and Environmental Microbiology. 2007 Mar 15;73(6):1712-1720

[11] Ruparelia JP, Chatterjee AK, Duttagupta SP, Mukherji S. Strain specificity in antimicrobial activity of silver and copper nanoparticles. Acta Biomaterialia. 2008 May 31;4(3):707-716

[12] Azam A, Ahmed AS, Oves M, Khan MS, Memic A. Size-dependent antimicrobial properties of $\mathrm{CuO}$ nanoparticles against Gram-positive and-negative bacterial strains. International Journal of Nanomedicine. 2012;7:3527 
[13] Azam A, Ahmed AS, Oves M, Khan MS, Habib SS, Memic A. Antimicrobial activity of metal oxide nanoparticles against Gram-positive and Gram-negative bacteria: A comparative study. International Journal of Nanomedicine. 2012;7:6003

[14] RisticBZ, Milenkovic MM, DakicIR, Todorovic-MarkovicBM, Milosavljevic MS, Budimir MD, Paunovic VG, Dramicanin MD, Markovic ZM, Trajkovic VS. Photodynamic antibacterial effect of graphene quantum dots. Biomaterials. 2014 May 31;35(15):4428-4435

[15] White JM, Powell AM, Brady K, Russell-Jones R. Severe generalized argyria secondary to ingestion of colloidal silver protein. Clinical and Experimental Dermatology. 2003 May 1;28(3):254-256

[16] Kwon HB, Lee JH, Lee SH, Lee AY, Choi JS, Ahn YS. A case of argyria following colloidal silver ingestion. Annals of Dermatology. 2009 Aug 1;21(3):308-310

[17] Israelachvili J. Intermolecular and Surface Forces. 2nd ed. San Diego, CA: Academic Press Inc; 1992

[18] Flemming HC, Neu TR, Wozniak DJ. The EPS matrix: The house of biofilm cells. Journal of Bacteriology. 2007 Nov 15;189(22):7945-7947

[19] Sutherland IW. Biofilm exopolysaccharides: A strong and sticky framework. Microbiology. 2001 Jan 1;147(1):3-9

[20] Flemming HC, Wingender J, Mayer C, Korstgens V, Borchard W. Cohesiveness in biofilm matrix polymers. In: Symposia-Society for General Microbiology 2000 Jan 1. Cambridge: Cambridge University Press; 1999. p. 87-106

[21] Kitazawa H, Toba T, Itoh T, Kumano N, Adachi S, Yamaguchi T. Antitumoral activity of slime-forming, encapsulated Lactococcus lactis subsp. cremoris isolated from Scandinavian ropy sour milk, viili. Animal Science and Technology (Japan). 1991; ISSN: 0021-5309

[22] Sheng X, Ting YP, Pehkonen SO. The influence of ionic strength, nutrients and $\mathrm{pH}$ on bacterial adhesion to metals. Journal of Colloid and Interface Science. 2008 May 15;321(2):256-264

[23] Ryu JH, Beuchat LR. Biofilm formation by Escherichia coli O157: H7 on stainless steel: Effect of exopolysaccharide and curli production on its resistance to chlorine. Applied and environmental microbiology. 2005 Jan 1;71(1):247-254

[24] Kang YS, Park W. Protection against diesel oil toxicity by sodium chloride-induced exopolysaccharides in Acinetobacter sp. strain DR1. Journal of Bioscience and Bioengineering. 2010 Feb 28;109(2):118-123

[25] Koutsoudis MD, Tsaltas D, Minogue TD, von Bodman SB. Quorum-sensing regulation governs bacterial adhesion, biofilm development, and host colonization in Pantoea stewartii subspecies stewartii. Proceedings of the National Academy of Sciences. 2006 Apr 11;103(15):5983-5988

[26] Christensen GD, Simpson WA, Younger JJ, Baddour LM, Barrett FF, Melton DM, Beachey $\mathrm{EH}$. Adherence of coagulase-negative staphylococci to plastic tissue culture plates: A 
quantitative model for the adherence of staphylococci to medical devices. Journal of Clinical Microbiology. 1985 Dec 1;22(6):996-1006

[27] Freeman DJ, Falkiner FR, Keane CT. New method for detecting slime production by coagulase negative staphylococci. Journal of Clinical Pathology. 1989 Aug 1;42(8):872-874

[28] Arciola CR, Campoccia D, Gamberini S, Cervellati M, Donati E, Montanaro L. Detection of slime production by means of an optimised Congo red agar plate test based on a colourimetric scale in Staphylococcus epidermidis clinical isolates genotyped for ica locus. Biomaterials. 2002 Nov 30;23(21):4233-4239

[29] Costerton JW, Montanaro L, Arciola CR. Biofilm in implant infections: Its production and regulation. The International Journal of Artificial Organs. 2005 Nov;28(11):1062-1068

[30] Mack D, Becker P, Chatterjee I, Dobinsky S, Knobloch JK, Peters G, Rohde H, Herrmann M. Mechanisms of biofilm formation in Staphylococcus epidermidis and Staphylococcus aureus: Functional molecules, regulatory circuits, and adaptive responses. International Journal of Medical Microbiology. 2004 Sep 24;294(2):203-212

[31] Patti JM, Allen BL, McGavin MJ, Hook M. MSCRAMM-mediated adherence of microorganisms to host tissues. Annual reviews in. Microbiology. 1994 Oct;48(1):585-617

[32] Speziale P, Pietrocola G, Rindi S, Provenzano M, Provenza G, Di Poto A, Visai L, Arciola CR. Structural and functional role of Staphylococcus aureus surface components recognizing adhesive matrix molecules of the host. Future Microbiology. 2009 Dec 8;4(10):1337-1352

[33] Hall-Stoodley L, Stoodley P. Biofilm formation and dispersal and the transmission of human pathogens. Trends in Microbiology. 2005 Jan 31;13(1):7-10

[34] Purevdorj B, Costerton JW, Stoodley P. Influence of hydrodynamics and cell signaling on the structure and behavior of Pseudomonas aeruginosa biofilms. Applied and Environmental Microbiology. 2002 Sep 1;68(9):4457-4464

[35] Hammer BK, Bassler BL. Quorum sensing controls biofilm formation in Vibrio cholerae. Molecular Microbiology. 2003 Oct 1;50(1):101-104

[36] von Götz F, Häussler S, Jordan D, Saravanamuthu SS, Wehmhöner D, Strüßmann A, Lauber J, Attree I, Buer J, Tümmler B, Steinmetz I. Expression analysis of a highly adherent and cytotoxic small colony variant of Pseudomonas aeruginosa isolated from a lung of a patient with cystic fibrosis. Journal of Bacteriology. 2004 Jun 15;186(12):3837-3847

[37] Yildiz FH, Schoolnik GK. Vibrio cholerae O1 el tor: Identification of a gene cluster required for the rugose colony type, exopolysaccharide production, chlorine resistance, and biofilm formation. Proceedings of the National Academy of Sciences. 1999 Mar 30;96(7):4028-4033

[38] Khalil H, Williams RJ, Stenbeck G, Henderson B, Meghji S, Nair SP. Invasion of bone cells by Staphylococcus epidermidis. Microbes and Infection. 2007 Apr 30;9(4):460-465

[39] Subbiahdoss G, Sharifi S, Grijpma DW, Laurent S, van der Mei HC, Mahmoudi M, Busscher $\mathrm{HJ}$. Magnetic targeting of surface-modified superparamagnetic iron oxide nanoparticles 
yields antibacterial efficacy against biofilms of gentamicin-resistant staphylococci. Acta Biomaterialia 2012 Jul 31;8(6):2047-2055

[40] Chong Y, Ma Y, Shen H, Tu X, Zhou X, Xu J, Dai J, Fan S, Zhang Z. The in vitro and in vivo toxicity of graphene quantum dots. Biomaterials. 2014 Jun 30;35(19):5041-5048

[41] Sun H, Gao N, Dong K, Ren J, Graphene QX. Quantum dots-band-aids used for wound disinfection. ACS Nano. 2014 Jun 2;8(6):6202-6210

[42] Habiba K, Bracho-Rincon DP, Gonzalez-Feliciano JA, Villalobos-Santos JC, Makarov VI, Ortiz D, Avalos JA, Gonzalez CI, Weiner BR, Morell G. Synergistic antibacterial activity of PEGylated silver-graphene quantum dots nanocomposites. Applied Materials Today. 2015 Dec 31;1(2):80-87

[43] Denkhaus E, Meisen S, Telgheder U, Wingender J. Chemical and physical methods for characterisation of biofilms. Microchimica Acta. 2007 Apr 1;158(1-2):1-27

[44] Wolf G, Crespo JG, Reis MA. Optical and spectroscopic methods for biofilm examination and monitoring. Reviews in Environmental Science and Biotechnology. 2002 Sep 1;1(3):227-251

[45] Neu TR, Swerhone GD, Lawrence JR. Assessment of lectin-binding analysis for in situ detection of glycoconjugates in biofilm systems. Microbiology. 2001 Feb 1;147(2):299-313

[46] Ritenberg M, Nandi S, Kolusheva S, Dandela R, Meijler MM, Jelinek R. Imaging Pseudomonas aeruginosa biofilm extracellular polymer scaffolds with amphiphilic carbon dots. ACS Chemical Biology. 2016 Feb 26;11(5):1265-1270

[47] Morrow JB, Holbrook RD. Association of quantum dot nanoparticles with biofilm. Journal of Environmental Quality. 2010 Nov 1;39(6):1934-1941

[48] Tenório DP, Andrade CG, Cabral Filho PE, Sabino CP, Kato IT, Carvalho LB, Alves S, Ribeiro MS, Fontes A, Santos BS. CdTe quantum dots conjugated to concanavalin A as potential fluorescent molecular probes for saccharides detection in Candida albicans. Journal of Photochemistry and Photobiology B: Biology. 2015 Jan 31;142:237-243

[49] Aldeek F, Mustin C, Balan L, Roques-Carmes T, Fontaine-Aupart MP, Schneider R. Surfaceengineered quantum dots for the labeling of hydrophobic microdomains in bacterial biofilms. Biomaterials. 2011 Aug 31;32(23):5459-5470

[50] Guisbiers G, Lara HH, Mendoza-Cruz R, Naranjo G, Vincent BA, Peralta XG, Nash KL. Inhibition of Candida albicans biofilm by pure selenium nanoparticles synthesized by pulsed laser ablation in liquids. Nanomedicine: Nanotechnology, Biology and Medicine. 2017 Apr 30;13(3):1095-1103 
